\title{
Experience of employment of computational models for water quality modelling
}

\author{
Anatoly Krutov ${ }^{1, *}$, Dilshod Bazarov ${ }^{2}$, Begzod Norkulov ${ }^{2}$, Bakhtiyar Obidov² and Bobur \\ Nazarov $^{2}$ \\ ${ }^{1}$ State Oceanographic Institute, Moscow, Russian Federation \\ ${ }^{2}$ Tashkent Institute of Irrigation and Agricultural Mechanization Engineers, Pumping stations, 100000 \\ Tashkent, Uzbekistan
}

\begin{abstract}
The purpose of the article is to develop the required and sufficient conditions under which numerical methods can be used for engineering calculations and for scientific research of hydrodynamic processes in solving practical problems related to predicting the spread of pollutants in water bodies and streams. The conducted studies consisted in comparing the results of laboratory experiments and mathematical modelling, in particular the distribution of heat in a stream with different temperature in water layers was studied. To check the adequacy of the proposed numerical models, calculations were performed and comparisons were made with the results of experimental data. The obtained results allowed to determine the boundaries of the qualitative difference in the flow behaviour for different numbers of Froude and Reynolds. The accuracy of the method was also studied. A number of additional requirements for numerical models were proposed in addition to approcsimation and stability, such as requirements of conservativeness (divergence), existence of trivial solutions on grids, possibility to calculate highly unsteady, quasi-stable, pulsating and stationary flows, requirement of invariance of linearized equations, as well as the requirement of a one-dimensional scheme to be a consequence of a twodimensional scheme. Distribution of velocities of wind currents using a three-dimensional and two-dimensional model was studied for a real object. A shallow-water bay of the Aral Sea was chosen as the object for the research. Comparison of the calculation results for both models showed that the flow velocity fields, as well as the distribution of pollutants in shallow waters, can be performed using a two-dimensional model.
\end{abstract}

*Corresponding author: krutov@bk.ru 


\section{Introduction}

Increase in the volume of use of water resources and contemporaneous increase of the requirements for the quality of water withdrawn set a number of tasks the solution of which can impact on the efficiency of the facilities and environmental safety. The most important of the emerging tasks are:

- determination of velocity fields in lakes and streams;

- determination concentrations of pollutants, including temperature and salinity in lakes and streams;

- prediction of bed flow deformations, roiling, and sedimentation;

- determination the degree of eutrophication of water bodies;

- development of engineering measures to regulate water quality both in water bodies and at water intakes;

- forecasting of water quality in water bodies.

The article considers options to cope with one of the mentioned above tasks as movement of concervative (nonpartitionable) pollutants in surface wates and consists of the following parts: basics on hydrodynamics equations used for mathematical modelling of hydraulic processes in water bodies, discussions of numerical models, major requirements for numerical models, comparison of the results of numerical calculations with experimental data, and conclusions.

\section{Basic equation}

In general, movement of pollutants in surface waters is an extremely complex combination of hydrochemical, hydrobiological and hydrodynamic processes. A complete picture of the quality of water cannot be described without taking into cjnsideration chemical and biochemical kinetics, sedimentation and diffusion of, for example, organic matter and a number of other physicochemical processes. In this paper, only hydraulic aspects of the movement of pollutants in surface water sources are considered and it is assumed that the interaction of chemical components occurs at a high rate, much higher than the propagation velocity of small perturbations $(\mathrm{c}=(\mathrm{gh}) 1 / 2+|\mathrm{U}|$, here $\mathrm{h},|\mathrm{U}|$ the depth and modulus of the flow velocity, respectively) and is described by first-order equations.

Sufficient accuracy is required for the design and operational practice as well as high efficiency of the methods to apply at engineering practice. These conditions are the prerequsits to solve problems while performing mass calculations of investigated objects assessing their current condition and forecast possible developments.

Currently, the most effective way of combining the mentioned conditions is mathematical modeling using the equations of hydrodynamics, which allows to identify the most important parameters necessary to obtain their numerical values for environmental protection practice.

The solution of the general equations of hydrodynamics is a complex problem and there are still no effective algorithms for solving three-dimensional equations in full definitions. However, there are solutions to overvome these difficulties by introducing some hypotheses. 
According to practice [1, 3 - 19, 30 - 32], for water badies whose horizontal dimensions are much greater than the depth, this can be done by introducing a large scale to consider the phenomenon of pollution movements. Depending on the degree of model upscaling, threedimensional baroclinic fluid equations, two-dimensional Saint-Venant equations, onedimensional equations and zero-dimensional equations (balance) could be considered.

In this paper, to study the capabilities of numerical models that reflect physical processes in water bodies and streams the following system of equations was used [2]:

$$
\left\{\begin{array}{l}
\frac{\partial u_{i}}{\partial t}+\frac{\partial u_{i} u_{j}}{\partial x_{j}}+\frac{\partial u_{i} w}{\partial z}+g\left(\frac{\partial z_{\Gamma}}{\partial x_{i}}+\frac{1}{\rho} \int_{z}^{z_{\Gamma}} \frac{\partial \rho}{\partial x_{i}} \partial z\right)=\frac{\partial}{\partial z} v_{T} \frac{\partial u_{i}}{\partial z}+\frac{\partial}{\partial z} \mu \frac{\partial u_{i}}{\partial z} \frac{1}{\rho} \\
\frac{\partial u_{i}}{\partial x_{i}}+\frac{\partial w}{\partial z}=0 \\
\frac{\partial S_{r}}{\partial t}+\frac{\partial S_{r} u_{j}}{\partial x_{j}}+\frac{\partial S_{r} w}{\partial z}=\frac{\partial}{\partial z} D \frac{\partial S_{r}}{\partial z}+q_{S z} \\
\rho=\rho\left(S_{r}\right)
\end{array}\right.
$$

here

$u_{i}$ - projection of the current velocity vector onto axis $x_{i}$,

$w$ - projection of the current velocity vector onto axis $\mathrm{z}$,

$\rho$ - liquid density,

$g$ - component of the acceleration vector of gravity force,

$v$ - kinematic coefficient of viscosity,

$D$ - coefficient of vertical diffusion,

$S_{r}$ - average substance concentration,

$q_{S r}$ - internal sources of a substance for the Newtonian fluid.

The essence of the method of finite differences consists in discretization of time and space variables (grid construction) and unknown functions (introduction of grid functions) from derivatives and integrals of these functions (construction of a difference scheme on the grid) [3 - 19]. As a result, instead of a system of differential or integral equations at each point of the considered area, a system of algebraic equations in each discrete structure (cell) of the considered area will be obtained.

\section{Theoretical preliminaries: Basic requirements for numerical models}

1. Conservatism (divergent). The requirement of conservatism is widely reflected in literature $[4-7,9-20,22-30]$, including the one related to the establishment of boundary conditions [2].

2. The scheme must have a trivial solution. On grids that do not change over time, the fulfillment of this requirement is not difficult. If, however, the grid on the real region changes with time, then this requirement leads to the necessity of introducing additional terms into the difference equations $[3,14,36]$.

3. The property of symmetry is necessary, i.e. if $U_{i}$ was changed by $-U_{i}$ in the area $1 \leq m \leq$ $M$ the the solution at points $m$ should be the same as before the replacement at points $M-m$ +1 .

4. For currents with small Froude number $(|U|<<\sqrt{g h})$ the difference scheme did not contradict the approximate self-similarity with respect to the Froude number [1, 3, 7 - 10, 30 - 33].

5. If the speed at the boundary is changed by a factor of $\alpha$, the velocities in the stationary flow (or time-averaged velocities in the pulsating flow) must also change by a factor of $\alpha$ [ 4 $-6,17,28,36]$. 
7. A one-dimensional scheme for straightened channels, taking into account the real change in the cross-flow area, would be a consequence of a two-dimensional one $[1,9,11,19]$.

The following conditions required for vodelling of pollutions transfer [2]:

1. Divergences (the caracteristic to accurately retaining the grid analogy of the impuritymass).

2. Invariance of the difference equations with respect to the transformation $\hat{S}=S+C$, here $\mathrm{S}$ - pollution concentration, $\mathrm{C}$ - constant,

3. Symmetry.

4. It is necessary to comply with the independence of the stationary state from time.

\section{Numerical models}

Following [8] complete equations of motion (without the hypothesis of hydrostatics):

$$
\begin{gathered}
\frac{\partial \Omega_{2}}{\partial t}-\left(\Omega_{1} \frac{\partial U_{2}}{\partial x_{1}}+\Omega_{2} \frac{\partial U_{2}}{\partial x_{2}}+\Omega_{3} \frac{\partial U_{2}}{\partial z}+\right)=\frac{\partial^{2} \tau_{1}}{\partial z}-\frac{\partial \rho}{\partial x_{1}} \frac{g}{\rho} \\
-\frac{\partial \Omega_{1}}{\partial t}-\left(\Omega_{1} \frac{\partial U_{1}}{\partial x_{1}}+\Omega_{2} \frac{\partial U_{1}}{\partial x_{1}}+\Omega_{3} \frac{\partial U_{1}}{\partial z}+\right)=\frac{\partial^{2} \tau_{2}}{\partial z}-\frac{\partial \rho}{\partial x_{2}} \frac{g}{\rho}, \\
\text { here } \Omega_{1}=\frac{\partial W}{\partial x_{2}}-\frac{\partial U_{2}}{\partial z} ; \Omega_{2}=\frac{\partial U_{1}}{\partial z}-\frac{\partial W}{\partial x_{1}} ; \Omega_{3}=\frac{\partial U_{2}}{\partial x_{1}}-\frac{\partial U_{1}}{\partial x_{2}} . .
\end{gathered}
$$

In the equations (4), it was assumed that the variation of the stresses along the horizontal coordinates is much smaller than along the vertical. Having made such an assumption about the flow velocities, the following was obtained from (4):

$$
\begin{gathered}
\frac{\partial \sigma_{1}}{\partial t}-\sigma_{1} \frac{\partial U_{2}}{\partial x_{2}}+\sigma_{2} \frac{\partial U_{1}}{\partial x_{2}}=\frac{\partial^{2} \tau_{1}}{\partial z^{2}}+g \frac{\partial \rho}{\partial x_{1}} \\
\frac{\partial \sigma_{2}}{\partial t}+\sigma_{2} \frac{\partial U_{1}}{\partial x_{1}}-\sigma_{1} \frac{\partial U_{2}}{\partial x_{1}}=\frac{\partial^{2} \tau_{2}}{\partial z^{2}}+g \frac{\partial \rho}{\partial x_{2}}, \\
\text { here } \tau_{1}=\mu \frac{\partial U_{1}}{\partial z} ; \tau_{2}=\mu \frac{\partial U_{2}}{\partial z} ; \mu=v \rho ; \sigma_{i}=\frac{\partial U_{i}}{\partial z} .
\end{gathered}
$$

In order to consider the task in the vertical limits independent of $x$, and $y$ a new coordinate system could be introduced:

$$
\widetilde{z}=\frac{z-z_{\text {Д }}}{z_{\Pi}-z_{\text {Д }}} ; \tilde{x}=x ; \tilde{y}=y ; \tilde{t}=t
$$

in which the flow area lies in the range from ' 0 ' to ' 1 '. In these coordinates, the equations have almost the same form as in the old coordinates, with $W$ replaced by $W_{0}$ :

$$
W_{0}=W-\frac{\partial h}{\partial t} \widetilde{z}-\left(\frac{\partial h}{\partial x_{1}} U_{1}+\frac{\partial h}{\partial x_{2}} U_{2}\right) \widetilde{z}-\left(\frac{\partial z_{\text {Д }}}{\partial x_{1}} U_{1}+\frac{\partial z_{\text {Д }}}{\partial x_{2}} U_{2}\right) .
$$

Henceforth, the sign “ " over variables is implied. 
In order to find fundamental approaches and cope with the task, let us assume that $\rho$ and $\mu$ are the functions which weakly depend on coordinates and time, and $\mu$ is the known (set) function. In addition, let us neglect the convective vertical transport in comparison with the diffusion. These assumptions do not affect fundamentals of the proposed approach. Moreover, a large number of tasks can be solved with these assumptions [2, 8].

Thus, under the assumptions made, we have the following system of equations:

With boundary conditions:

$$
\begin{aligned}
& \frac{\partial \tau_{1}}{\partial t}+U_{j} \frac{\partial \tau_{1}}{\partial x_{j}}-\tau_{1} \frac{\partial U_{2}}{\partial x_{2}}+\tau_{2} \frac{\partial U_{1}}{\partial x_{2}}=\frac{\mu}{h^{2}} \frac{\partial^{2} \tau_{1}}{\partial z^{2}} \\
& \frac{\partial \tau_{2}}{\partial t}+U_{j} \frac{\partial \tau_{2}}{\partial x_{j}}+\tau_{2} \frac{\partial U_{1}}{\partial x_{1}}-\tau_{1} \frac{\partial U_{2}}{\partial x_{1}}=\frac{\mu}{h^{2}} \frac{\partial^{2} \tau_{2}}{\partial z^{2}} \\
& \tau_{1}=\frac{\mu}{h} \frac{\partial U_{1}}{\partial z} \\
& \tau_{2}=\frac{\mu}{h} \frac{\partial U_{2}}{\partial z} \\
& \frac{\partial U_{i}}{\partial x_{i}}+\frac{1}{h} \frac{\partial W}{\partial z}=0
\end{aligned}
$$

$$
\begin{gathered}
\left.U\right|_{z=0}=\left.0 \vee U\right|_{z=0}=\sqrt{\frac{\tau_{b}}{\alpha_{b}}} \\
\left.\vec{\tau}\right|_{z=1}=\vec{\tau}_{n}=\lambda W_{i}|W| \\
\frac{\partial z_{s}}{\partial t}+\left(U_{s}\right)_{i} \frac{\partial z_{s}}{\partial x_{i}}=W_{s}
\end{gathered}
$$

Obviously, conditions (6.1) - (6.3) are not sufficient. In fact, as reported in [21], this is the main problem when calculating a turbulent flow. This problem is referred in the literature as the 'enclosure problem'.

One more condition is necessary. However, there are no physical prerequisites for its setting. This happened because by moving to the equations in 'stresses', we eliminated 'pressure', i.e. the additional condition is laid down in the original statement of the task. Indeed, integrating the first two equations (1), the missing condition could be obtained:

$$
\begin{array}{r}
\rho\left(\frac{\partial \bar{U}_{i} h}{\partial t}+\frac{\partial \bar{U}_{i} \bar{U}_{j} h}{\partial x_{j}}\right)+\rho g h \frac{\partial z_{\Pi}}{\partial x_{i}}+\frac{\partial R_{i j} h}{\partial x_{j}}=\left(\tau_{\Pi}-\tau_{\text {Д }}\right)_{i} \\
i=1,2 \\
\text { here: } R_{i j}=\int_{z_{\text {д }}}^{z_{\Pi}} \rho\left(U_{i}-\bar{U}_{i}\right)\left(U_{j}-\bar{U}_{j}\right) d z ; \bar{U}=\frac{1}{h} \int_{z_{\text {म }}}^{z_{\Pi}} U_{i} d z .
\end{array}
$$

With the introduction of equations averaged over the depth, the enclose problem is solved. In this case, it turns out to be more convenient instead of condition (6.3) to use the averaged equation of continuity:

$$
\frac{\partial h}{\partial t}+\frac{\partial \bar{U}_{i} h}{\partial x_{i}}=0
$$


An algorithm for solving equations (5) for flows with high viscosity using Chebyshev polynomials was proposed in [2]. In [2], numerical experiments were carried out to investigate the accuracy of the method in the following way: for a given number of operations $M=n N$ various ratios of the number of layers ' $n$ ' were taken and the number of polynomials ' $N$ ':

1. $n=1, N=21$

2. $n=7, N=3$

using the heat equation:

$$
\frac{\partial u}{\partial t}=\mu \frac{\partial^{2} U}{\partial z^{2}}
$$

with the initial condition $\left.U\right|_{t=t_{0}}=\delta\left(t-t_{0}\right)$ in the point $\mathrm{z}=0$, a numerical solution was compared with the analytical one.

Numerical tests shown that in all cases there is an over time tendency of the numerical solution to the exact one [2]. The best matches with the analytical solution, compared with other options, were obtained with $n=7, N=3$.

The diffusion of $\delta$-function on the interval [-1-1] could be used as an the example of such an over time-varying solution of the heat equation:

$$
U(t)=\frac{e^{-Z^{2} / 4 a t}}{2 \sqrt{\pi a t}} \quad(a=\mu=1)
$$

This serves as a good illustration of the advantages of solving it with the help of polynomials as well as the feasibility of splitting into layers with the representation of the function by the number of polynomials $N=3$ [2].

There was also a series of numerical experiments for $N=3(n=7)$ with different variants of equations for determining $a_{0}^{j}, a_{1}^{j}, a_{2}^{j}(j=\overline{1, n})$. It was shown that the best option is to use a quadratic polynom in combination with the Galerkin method [2].

One of the serious problems encountered in the prediction of the spread of pollutants is the problem of adequacy of simulated results to actual processes. In this respect, the only criterion is the comparison of calculations with actual observations.

\section{Comparison of the results of numerical calculations with experimental data}

A large number of studies were devoted to the problem of adequacy of the models which describe hydrodynamic phenomena in water bodies, for example [10 - 16, 26 - 30,25-27].

To verify the adequacy of the proposed models, calculations were performed and comparisons were made with the results of model studies published in [34].

To analyse the effects of stratification on flow behaviour, velocity and density measurements were taken under different flow conditions. The techniques used for these measurements are explained below.

The essence of the experiment [34] was as follows: the rectangular channel consisted of a $50.0 \mathrm{~m}$ long flume, whose first $0.5 \mathrm{~m}$ were adapted to create a two layer stratified flow. A separate supply of two flows with different temperatures moving at different speeds was arranged by two inlets. The height of each inlet was $0.1 \mathrm{~m}$ (Fig.1).

After $0.5 \mathrm{~m}$ the channel opened and the fluids from the two ducts started to mix. The depth of the flow was controlled by an outlet weir to obtain uniform flow with a flow depth of 0.2 
m. These dimensions classify the channel as a narrow channel, with aspect ratio (BIH) of 1 , and relative length (LIDh) of 100.

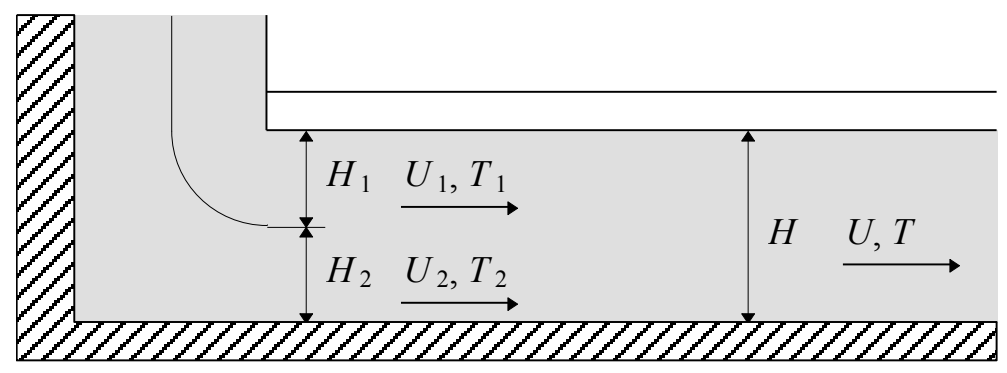

Fig. 1. Scheme of the model.

The average temperature was measured continuously using a platinum resistance of the following dimensions: length $-1.5 \mathrm{~cm}$, width $-0.3 \mathrm{~cm}$, connected to secondary equipment. The average velocity was measured by micro-curent meter of $0.9 \mathrm{~cm}$ diameter every $2 \mathrm{~cm}$ from the bottom to the surface. The averaging time was $100 \mathrm{~s}$.

The mean square deviation of the temperature fluctuations was measured through each $1 \mathrm{~cm}$ of the profile using a heated wire sensor with dynamic feedback and integrator, which allowed to catch information at frequencies from 0.2 to 10 hertz. This seems sufficient for the experiment as these frequencies are the most important.

The effect of the following independent parameters was studied:

$$
\begin{gathered}
\operatorname{Re}=\frac{U_{1} h}{v}-\text { Reynolds number } \\
\operatorname{Fr}=\frac{{ }_{\Delta} U}{\sqrt{g_{\Delta} \rho h}}-\text { modified Froude number, }
\end{gathered}
$$

Both Reynolds number and Froude number changed in the experiment in the following intervals:

$$
\begin{aligned}
& 5 \cdot 10^{3} \leq \operatorname{Re} \leq 10^{4} \\
& 0,9 \leq \mathrm{Fr} \leq 5 \\
& \frac{U_{2}}{U_{1}}=2 \text { and } 3
\end{aligned}
$$

here ${ }_{\Delta} \mathrm{U}$ - velosity difference, ${ }_{\Delta} \rho$ - difference between densities of two flows, $v$ - molecular viscosity.

Experiments allowed to establish boundaries of the qualitative difference in the behavior of the flow at various Froude and Reynolds numbers. It was found that at $\mathrm{Fr}>1,6$ there is a transition from a stable stratification to an unstable.

Fig. 2 illustrates the comparison of the experimental data and the numerical implementation of the same model for two Reynolds $\left(5^{*} 103\right.$ and $\left.1 * 10^{4}\right)$ and Froude (0.9 and 5.0) numbers.

As a result of the experiment, vertical profiles of mean temperature $(T)$, average horizontal speed (U) and mean-square deviation of the temperature fluctuation amplitude were identified. 

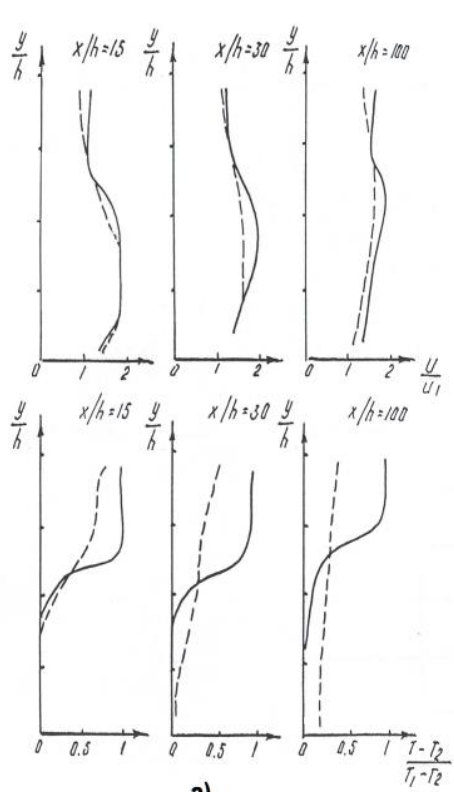

a)
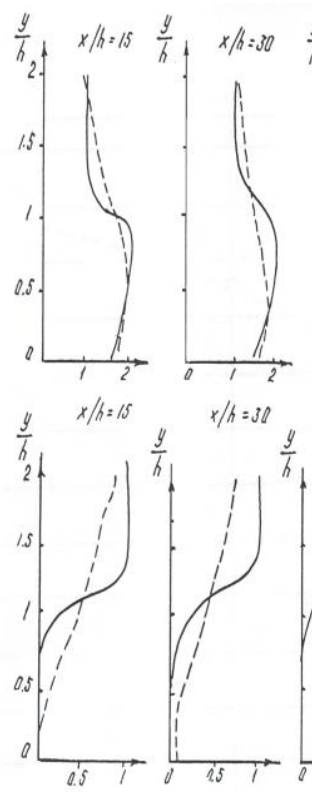
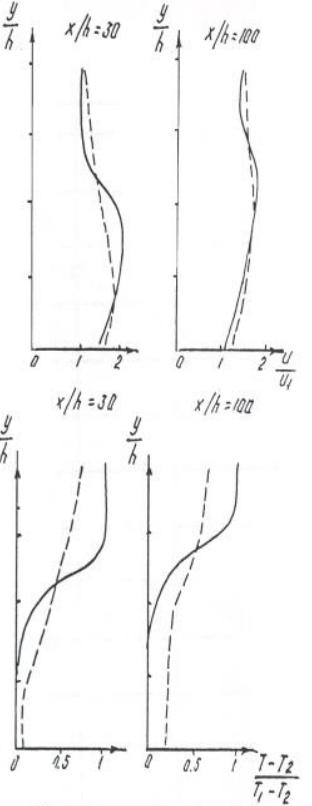

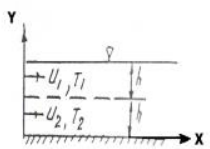

Legend

a) $R_{e}=5 \cdot 10^{3} ; F_{2}=0.9$

b) $\quad R e=10^{4} ; F_{2}=5,0$

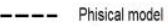

b)

Fig. 2. Comparison of experimental data and numerical modelling.

As can be seen from the figures, the velocity and temperature of water, determined by calculations, best of all coincide with the experimental results for case a), i.e. under $\operatorname{Re}=5 *$ $10^{3}$ and $\mathrm{Fr}=0.9$. In this case, the maximum deviation of the calculated water velocity on the surface from the one measured does not exceed $0.20 \mathrm{U} / \mathrm{U}_{1}$ in the whole experimental area, and at the level $\mathrm{y} / \mathrm{h}=0.9$ at a distance $\mathrm{x} / \mathrm{h}=30$ it was $0.25 \mathrm{U} / \mathrm{U}_{1}$.

In case b), i.e. under $\operatorname{Re}=1 * 10^{4}$ and $\mathrm{Fr}=5.0$, the maximum deviation of the calculated water velocity from the measured one on the water surface does not exceed $0.05 \mathrm{U} / \mathrm{U}_{1}$, and at the level of $0.8 \mathrm{y} / \mathrm{h}$ does not exceed $0.25 \mathrm{U} / \mathrm{U}_{1}$. The maximum deviation of the calculated water velocity was fixed at a distance $\mathrm{x} / \mathrm{h}=30$ at a depth of $0.9 \mathrm{y} / \mathrm{h}$. At this point, the deviation was $0.34 \mathrm{U} / \mathrm{U}_{1}$.

At a distance $\mathrm{x} / \mathrm{h}=100$, the coincidence of the flow rates obtained in the experiment and as a result of the calculations turned out to be good in both a) and b). The proposed model better described the temperature distribution in the flow with $\mathrm{Fr}=5.0$. Most likely this is due to the fact that the model describes the turbulent flows fairly well.

The problem of adequacy lies not only in comparison of the results of calculations with the data of experimental studies but to a large extent in the complexity of conducting both laboratory and full-scale experiments [29]. From this point of view, the results of the comparisons given in $[9,2]$ show that models similar to (1) are in a good agreement between the results of measurements and calculations.

A good agreement between the experimental data published in [27, 28] and the numerical implementation suggests that the proposed model describes turbulent flows well and can be used for numerical modelling of objects with different-density flows.

Comparison of acual wind borned current in the channel in physical model [34] and calculations were made to of determine the adequacy of the proposed model. The result of the comparison is presented in Figure 3. 


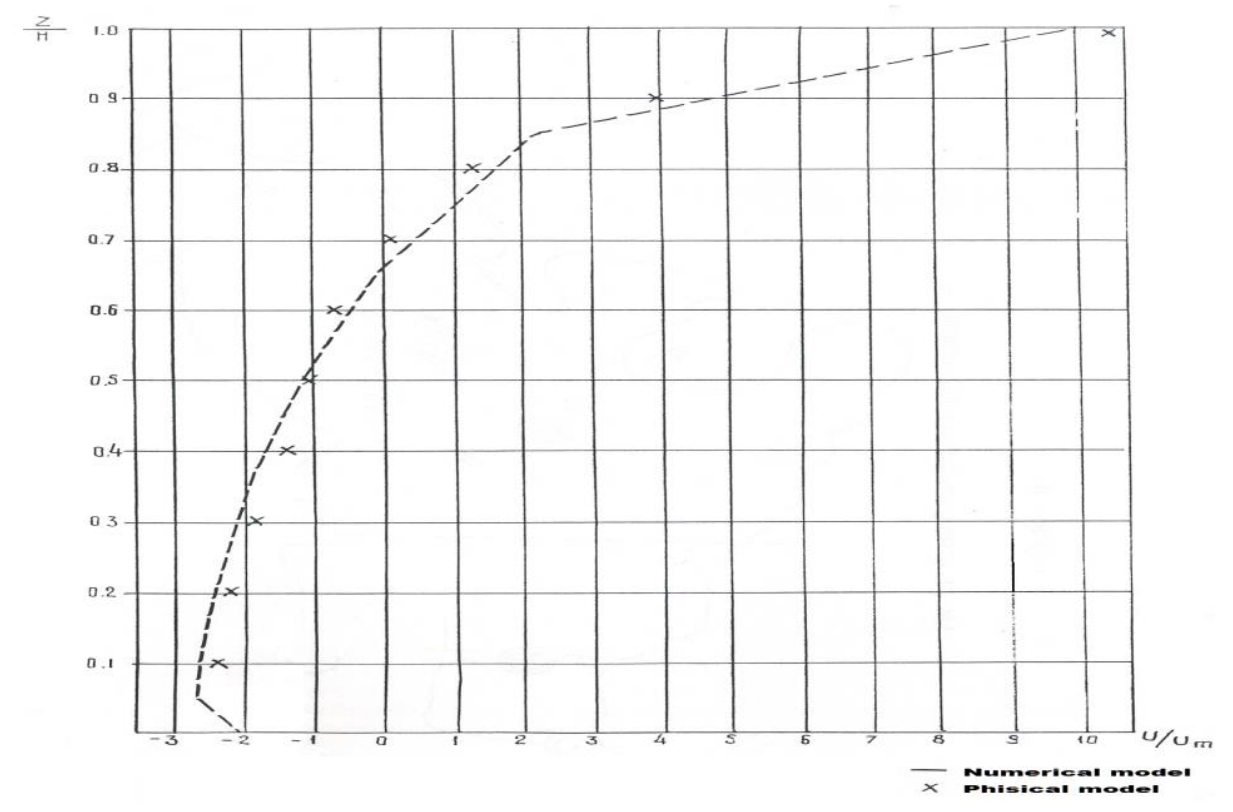

Fig. 3. Comparison of experimental data and numerical modeling for the wind current in the channel.

The results of detailed studies of the properties of the model (5) and a comparison of the results of calculations using other models are given in 27. Comparisons made showed good agreement with the results of calculations using the proposed model.

\section{Conclusions}

The accumulated practical experience of applying finite-difference schemes for the solution of dynamic tasks made it possible to find the necessary conditions under which numerical methods can be used for engineering calculations and studies of hydrodynamic processes.

To solve practical problems associated with predicting the muvement of pollutants in water bodies and streams, a grid-spectral method was developed that showed high efficiency in the case of stratified currents. This is especially justified for solving problems associated with the calculation of stratified flows. An algorithm based on the use of integro-interpolation methods was developed. The algorithm allows to carry out calculations with large time steps. Numerical studies using a three-dimensional model of flow and dispersal of pollutions in the investigated reservoir showed that, in general, the velocity fields at different depth levels are identical, i.e. the influence of three-dimensionality is insignificant. Therefore, it is advisable to carry out numerical modeling of the distribution of pollutions across the water area of reservoirs with similar characteristics using a two-dimensional (plan) model.

Numerical simulation using a two-dimensional model gave a picture of the velocity and impurity fields over the area of the reservoir in the dynamics of development over a period of 5 years as well as the field of maximum values of pesticide $\gamma-\mathrm{HCH}$.

\section{Reference}

1. V.M. Lyather, A.N. Militeev, Numerical modeling of open streams. Water Resources. (1987) .1. C.35-41. 
2. A.N. Krutov, Development of the theory and methods of forecasting the spread of pollutants in the surface waters of arid zones. Dis. on the competition scholarly step. Doctor of Technical Sciences, M. (1997), 162 p.

3. A.N. Militeev, Solving problems of hydraulics of small reservoirs and pools of waterworks using numerical methods. Author's abstract diss. doctors tech. Sciences.- M., (1982). 43c

4. F.F. Vasiliev, T.A. Temnoeva, S.M. Shugrin, A numerical method for calculating unsteady currents in open channels. Ed. USSR Academy of Sciences. mechanics, (1965)

5. O. F. Vasiliev, M.T. Gladyshev, On the calculation of discontinuous waves in open channels. Izv. Academy of Sciences of the USSR, ser. Fluid and gas mechanics, (1966) 6. P. 184-189.

6. O. F. Vasiliev, A.F. Voevodin, A.A. Atavin, Methods for calculating unsteady currents in open channel systems and channels. Numerical methods of continuum mechanics, (1975). V. 6. No. 4. P. 21-30.

7. D.V. Kvon, Mathematical modeling of hydrothermal processes in Lake Teletskoye. Author's abstract dis. on the competition scholarly step. Ph.D. sciences. Barnaul. (1998) $21 \mathrm{p}$.

8. A.N. Militeev, D.R. Bazarov, A.N. Krutov, Three-dimensional mathematical model for streams with a blurred bottom. Messages in applied mathematics. M., the CC of RAS, (1997) $45 \mathrm{p}$.

9. V.M. Lyather, A.N. Militeev, Hydraulic investigations by numerical methods. Water resources. (1981) 3. S.60-79.

10. A.N. Militeev, Numerical modeling of pulsation currents and heat and mass transfer in small unstratched water bodies. Hydraulic research in the energy and water sector. - M., (1983) P.8-22.

11. M.G. Gladyshev, Numerical modeling of unsteady currents in open channels. Water resources. (1981) 3. C.119-125.

12. O.F. Vasilyev, G.V. Volkova, V.I. Kvon, Numerical calculation of the hydrothermal mode of cooling ponds of thermal power plants and nuclear power plants. Tr. coordinates owls on hydraulic engineering., Energy, - L., (1977) 115. C. 59-64.

13. O.F. Vasiliev, M.G. Gladyshev, On the calculation of discontinuous waves in open channels. Izv. Academy of Sciences of the USSR. Fluid and gas mechanics. (1966) 6. C. 53-61.

14. O.F. Vasilyev, M. G. Gladyshev, VGSudobicher, Numerical calculation of unsteady water movement in an open channel. The solution of one-dimensional problems of gas dynamics in moving grids. M .: Science, (1970) C. 48-61.

15. M.G. Gladyshev, Numerical modeling of unsteady currents in open channels. Water resources. (1981) 3. C.119-125.

16. V.I. Kvon. Hydrothermal calculation of cooling ponds. Izv. Academy of Sciences of the USSR. Energy and transport. (1979). 5.C.49-60

17. B.L. Istorik, Numerical research of concretional flows in open channels. Sat scientific Trudov. Hydroproject them. S.Y. Beetle Hydraulics and filtration. M., (1979). C.18-28.

18. B.L.Istorik, V.M. Lyather, Propagation of breakthrough waves in a prismatic channel.Izv. Academy of Sciences of the USSR. MZHG. (1975). 1. P.39-42.

19. A.S. Petrusev, Difference schemes and their analysis: a teaching aid. M .: MIPT, (2004). $89 \mathrm{~s}$.

20. A.N. Militeev, N.V. Ovinova, Method of calculating stratified reservoirs. Inst. ZNIIS. M., (1993) 14 p .- Dep. in VNIINTI 03.11.93, 2744-B93.

21. W. Rodi, (1993) Turbulence - A state-of-the-art review. IAHR Monograph, Third Edition, A. A. Balkema, Rotterdam, Brookfield. 
22. A.N. Militeev, Yu.B.Shpolyansky Numerical Modeling of Pollution Transfer by Design of Water Protection Measures. Optimization of technological processes and improvement of design based on hydraulic research. M., (1989). P.46-52

23. I.A. Sherenkov, Applied planned problems of the hydraulics of quiet flows .- M .: Energy, (1978). 240s

24. I.Shiryak, Investigation of the transport of pollutants in shallow-flowing water bodies by wind currents: Author's abstract. diss. Cand. tech. Sciences.- L., (1978). 23c.

25. A.T. Zinoviev, K.B. Koshelev, K.V. Marusin, E.D. Kosheleva. Mathematical modeling of the channel flow to predict the impact of construction in floodplains on the hydrological regime of large rivers (using the Ob River as an example). Russian Water Management No 2, 2017 Available from: https://www.researchgate.net/publication/318851108 [accessed Mar.22.2019].

26. Potapov I. I., Snigur KS. Modeling the evolution of the sandy-gravel bottom of the channel in a one-dimensional approximation // Computer Research and Modeling. 2015.T.7 № 2.S.315-329.

27. Belikov V.V., Borisova N.M., Gladkov G.L. Mathematical model of sediment transport for calculating the record of dredging slots and channel open pits. // Bulletin of the State University of Maritime and River Fleet. Admiral S.O. Makarova. Journal of the University of Water Communications. 2010. Issue 2., pp. 105 - 113.C.F. Scoit, (1994) A numerical study of the interaction of tidal oscillation and nonlinearities in an estuary. Estuarine, Coastal and Shelf Science, 39, pp. 477-496.

28. B.E. Launder, and W.M. Ying, (1973) Prediction of flow and heat transfer in ducts of square cross-section. Proceedings of the Institution of Mechanical Engineers. 187, pp. 455-461.

29. B.Lin, and K.Shiono, (1992) Prediction of pollutant transport in compound channel flows. Proceedings of the Second International Conference on Hydraulic and Environmental Modelling of Coastal, Estuarine and River Waters, Ashgate Publishing Limited, 2, pp. 373-384.

30. Hydraulic Research Station (1974). The wash water storage scheme numerical model studies of the Great Ouse Estuary. Report DE 11, Wallingford, UK.

31. D.Cokijat, (1991) Mathematical modelling of turbulent flows in two stage channels. Transfer Report, Proceedings of the Hydraulics Division, Department of Civil Engineering, City University, London, UK, pp. 1-78.

32. A.F. Blumberg, (1977) Numerical model of estuarine circulation. Journal of Hydraulics Division, Proceedings of the American Society of Civil Engineers, 103, HY3, pp. 295310.

33. P.L. Violett, Turbulent mixing in a two layer stratified shear flow. Seond Int. Symposium on stratified flow.- Troundheim, Norway. (1980). Pp.315-325.

34. Renato do Nascimento Siqueira, (2002). Transport and mixing processes in stratified flow. Loughborough University Institutional Repository. Retrieved 17.12.2018 from https://dspace.lboro.ac.uk/dspace-jspui/bitstream/2134/34335/1/Thesis-2002-

DoNascimentoSiqueira.pdf

35. M.B. Abbot, D.R. Basco, Computational Fluid Dynamics. An Introduction for Engineers. Copublished in the United States with John Wiley and Sons, Inc. New York, (1994), pp.425.

36. W.D. Baines, D.I. Knapp Wind driven water current. J. Hydraulics Division. ASCE. (1965).-91. P.821-836. 